\title{
Pandemiebekämpfung in der deutschen Gesundheitspolitik
}

\author{
Karin Knufmann-Happe
}

\begin{abstract}
„Die Pandemie, die keine war“, so lautet der Titel des Leitartikels vom 4. Januar 2010 in der Welt Berlin. War es keine? Die angesichts des weiterhin moderaten Verlaufs der A/H1N1-Pandemie einsetzende Diskussion darüber, ob die sog. Schweinegrippe eine „echte Pandemie“ ist, ebenso wie die an die Weltgesundheitsorganisation (WHO) gerichteten Forderungen, die Pandemiedefinition zu ändern und der Krankheitsschwere größeres Gewicht beizumessen, geben Anlass auf einige Aspekte dieses für die Pandemiebekämpfung zentralen Begriffs einzugehen.
\end{abstract}

\section{Pandemie}

Die WHO spricht von einer Pandemie, wenn sie in mindestens zwei WHORegionen eine wachsende und anhaltende Mensch-zu-Mensch-Übertragung eines Influenzavirus feststellt, gegen das die Bevölkerung keine oder nur eine eingeschränkte Immunität entwickelt hat.

Diese Situation war am 11. Juni 2009 eingetreten. Im April 2009 war A/H1N1/2009 von den Centers for Disease Control der USA erstmals als neue Virusvariante klassifiziert worden. Am 25. April 2009 erklärte die WHO offiziell den Notfall für die öffentliche Gesundheit von internationaler Bedeutung (public health emergency of international concern) und erhöhte innerhalb der folgenden vier Tage die Pandemiewarnstufe von 3 auf 5. Das Virus war zunächst in Nordamerika (Kalifornien), Mexiko und Kanada identifiziert worden und hatte sich im Verlauf des Mai rasch weltweit ausgebreitet. Als die WHO Anfang Juni das Erreichen der Pandemiephase 6 erklärte, waren in 74 Ländern weltweit rund 30.000 Infektionen mit dem neuen Influenzavirus registriert worden.

Pandemie bezeichnet demnach ein sich global ausbreitendes Krankheitsgeschehen, das unterschiedliche Regionen $\mathrm{zu}$ unterschiedlichen Zeitpunkten in ganz unterschiedlicher Weise betreffen kann. Die Schwere der Erkrankung war und ist bislang kein Kriterium für die Heraufsetzung der WHO-Pandemiestufen. Sie ist jedoch selbstverständlich ein wesentlicher Faktor wenn es darum geht, lageangepasst Eingrenzungs- und Bekämpfungsmaßnahmen zu ergreifen. Entsprechend hat die WHO zeitgleich mit der Ausrufung der Pandemie eine Einschätzung zur klinischen Symptomatik veröffentlicht, in der sie die Pandemie als „derzeit moderat" charakterisiert hat. 
Ausbreitung, Dynamik und Schwere einer Pandemie sind auf der Basis der uns heute zur Verfügung stehenden Erkenntnisquellen nur sehr eingeschränkt voraussagbar. Zugleich haben Pandemien das Potential, die Gesundheit der Bevölkerung, das Wirtschaftsleben und im Extremfall das gesellschaftliche Zusammenleben insgesamt erheblich zu beeinträchtigen.

Die jeder Pandemie innewohnenden Risiken machen auch unter Vorsorgegesichtspunkten spezifische Maßnahmen erforderlich, zumal einige der möglichen Bekämpfungsmaßnahmen einen nicht unerheblichen zeitlichen Vorlauf benötigen. Dies gilt insbesondere für die Impfstoffentwicklung und -produktion.

\section{Pandemieplan}

Außerhalb der Fachöffentlichkeit kaum wahrgenommen, reicht die Pandemieplanung in Deutschland schon in das Jahr 2001 zurück, als am Robert KochInstitut eine Expertengruppe Influenzapandemieplanung ihre Arbeit aufnahm. 2005 wurde dann der Nationale Pandemieplan veröffentlicht; im Mai 2007 erschien eine aktualisierte Fassung. Von Anbeginn an wurde nicht allein der Gesundheitsbereich in den Blick genommen, sondern alle potentiell betroffenen staatlichen Stellen und darüber hinaus Wirtschaftsbetriebe und die Zivilgesellschaft.

Mit der beim Robert Koch-Institut (RKI), einer Fachbehörde im Geschäftsbereich des Bundesministeriums für Gesundheit, einberufenen Influenzakommission wurden zentrale Vertreter der Fachöffentlichkeit und der Verbände intensiv an der Pandemieplanung beteiligt und auch in die laufende Pandemiebekämpfung eingebunden.

Breitere Aufmerksamkeit in der allgemeinen Öffentlichkeit erlangte die Pandemieplanung erstmals mit dem Auftreten der Vogelgrippe und im Zusammenhang mit der 2007 durchgeführten Stabsrahmenübung LÜKEX, der ein Pandemieszenario zugrunde lag.

Pandemiepläne erfüllen komplexe Funktionen. Aus der Sicht der Gesundheitspolitik sind drei Aspekte besonders hervorzuheben:

1. Pandemieplanung entwickelt und beschreibt die Strategie der Bekämpfung potentieller, großflächiger und grenzüberschreitender gesundheitlicher Bedrohungslagen. Sie schafft Transparenz über Strategie und mögliche Maßnahmen für Verantwortungsträger in Bund, Ländern und Kommunen, Fachöffentlichkeit und Öffentlichkeit. Beides ist nicht nur zentrale Voraussetzung für eine angemessene Vorbereitung auf eine Pandemie, sondern auch für eine erfolgreiche Bewältigung. 
2. Pandemiepläne tragen der Notwendigkeit eines bereichs- und ebenenübergreifenden Zusammenwirkens der Verantwortungsträger in Bund, Ländern und Kommunen und darüber hinaus in Wirtschaft und Gesellschaft Rechnung. Sie sind damit weit mehr als ein Hilfsmittel, um den Herausforderungen des föderalen Staatsaufbaus mit seiner verfassungsrechtlichen Aufgabenzuweisung und einem pluralistischen, von Selbstverwaltung geprägten Gesundheitswesen zu begegnen.

3. Pandemiepläne unterstützen eine flexible und gleichwohl strukturierte Erfüllung komplexer Aufgaben auf unterschiedlichen Handlungsebenen (international, europäisch und national) durch Bund, Land und Kommune, Gesundheitseinrichtungen, Wirtschaftsunternehmen, Nichtregierungsorganisationen. Voraussetzung ist, dass die Planungen die erforderliche Flexibilität aufweisen, damit auch bei sehr unterschiedlichen Lagen angemessen agiert wird.

Aufbauend auf dem Nationalen Pandemieplan, auf den sich Bund und Länder verständigt haben und auf die umfangreichen fachlichen Empfehlungen, die in diesem Kontext erarbeitet und veröffentlicht wurden, haben Länder und Kommunen aber auch die Einrichtungen im Gesundheitswesen sowie weitere öffentliche und private Unternehmen eigene Pandemiepläne erstellt. Hier sind spezifische Detailplanungen niedergelegt. Diese gestufte Herangehensweise ermöglicht es, den jeweiligen Besonderheiten wie etwa regionalen Unterschieden in der Arztdichte oder der Ausstattung des öffentlichen Gesundheitsdienstes Rechnung zu tragen, die selbst auf der gleichen Handlungsebene sehr unterschiedliche Herangehensweisen erforderlich machen können.

Voraussetzung dafür, dass auf der Grundlage dieser Planungen wirkungsvolle Pandemiebekämpfung gelingt, ist das sinnvolle Ineinandergreifen nicht nur der nationalen Maßnahmen, sondern auch ein international koordiniertes Vorgehen. Hier kommt der WHO die entscheidende Rolle zu, aber auch die EU-Ministerräte, die Europäische Kommission und die Europäischen Agenturen leisten ihren spezifischen Beitrag.

\section{Herausforderungen für die Gesundheitspolitik}

Pandemien konfrontieren Gesundheitspolitik mit einem prozesshaften Geschehen. Sie muss in dieser Lage vielfach weitreichende Entscheidungen unter großer Unsicherheit treffen:

Gesundheitspolitik muss damit umgehen, dass sich die Lage jederzeit verändern kann - etwa wenn sich durch Mutation des Virus Resistenzen bilden und die Erkrankung nicht länger erfolgreich medikamentös behandelbar ist oder dass sie 
sich anders entwickelt als erwartet - wenn etwa die produzierten Impfstoffmengen hinter den antizipierten Mengen zurückbleiben.

Sie muss auch damit umgehen, dass neue Erkenntnisse über die Erkrankung nur sukzessive gewonnen werden. So lagen beispielsweise in der aktuellen Pandemie belastbare Erkenntnisse über das Erfordernis einer nur einmaligen Impfung erst zu einem so späten Zeitpunkt vor, dass einige unserer europäischen Nachbarn bereits Impfstoff in einem Umfang geordert hatten, der eine zweimalige Durchimpfung der jeweiligen Bevölkerung ermöglicht hätte. Ein zu langes Zuwarten birgt das Risiko, dass eine geeignete Bekämpfungsmaßnahme zu spät kommt. Eine als Überreaktion wahrgenommene Entscheidung kann die Akzeptanz in der Bevölkerung erheblich beeinträchtigen.

Pandemie ist ein Geschehen, bei dem sich Gesundheitspolitik in Bund und Ländern in hohem Maße auf wissenschaftliche Beratung stützen muss. Die Glaubwürdigkeit der wissenschaftlichen Fachbehörden auf internationaler und europäischer Ebene aber vor allem auch national, ihre hohe Fachlichkeit und die Unabhängigkeit sind für die Pandemiebekämpfung essentiell. Die Gesundheitspolitik in Bund und Ländern wurde seit Ausbruch der aktuellen Pandemie engmaschig und kontinuierlich insbesondere vom Robert Koch - und vom Paul Ehrlich - Institut beraten und hat ihre Entscheidungen auf die fachlichen Empfehlungen der Institute, die Entscheidungen der WHO und der Europäischen Arzneimittelbehörde bzw. der Kommission gestützt. Die Erfahrung zeigt: In der Pandemie übernehmen die wissenschaftlichen Institute eine tragende Rolle, nicht nur in der Politikberatung, sondern insbesondere auch bei der Informationsvermittlung an die Fachöffentlichkeit und die allgemeine Öffentlichkeit.

Eine Pandemie bringt höchste Anforderungen an die Krisenkommunikation mit sich. Der Erfolg der Pandemiebekämpfung hängt in hohem Maße davon ab, dass die von den Behörden verordneten oder empfohlenen Maßnahmen in der Bevölkerung auf Akzeptanz stoßen und befolgt werden. Dies gilt insbesondere, solange die Schwelle zu Zwangsmaßnahmen nicht überschritten ist. Diese Schwelle liegt in Deutschland zu Recht sehr hoch, insbesondere wenn es um Eingriffe in die körperliche Unversehrtheit geht (Impfpflicht). Um so wichtiger sind die verlässliche Information und Aufklärung der Bevölkerung. In einer sich dynamisch entwickelnden Pandemie kann dies in großem Umfang nur durch intensive 'Pressearbeit' der Behörden in Bund und Ländern geleistet werden.

\section{Aufgabe der Gesundheitspolitik in einer Pandemie}

Die zentrale Aufgabe der Gesundheitspolitik in einer Pandemie ist der Schutz der Gesundheit der Bevölkerung. 
Dabei steht außer Frage, dass eine Pandemie je nach Verlauf über den Bereich der Gesundheit hinaus erhebliche Auswirkungen auf Wirtschaft und Gesellschaft haben kann. Gesundheitspolitik ist selbstverständlich auch hier gefordert. Sie muss rechtzeitig auf die Gefahren hinweisen. Sie muss kontinuierlich verlässliche Information bereitstellen und Empfehlungen geben und notwendige Gegenmaßnahmen initiieren. Richtig war es daher, dem Gesundheitsschutz im Zivilschutz- und Katastrophenhilfegesetz 2009 einen größeren Stellenwert zu geben.

Pandemie beschreibt ein weltumspannendes Geschehen. Wir haben hier ein vitales Interesse am Funktionieren der internationalen Zusammenarbeit. Eine robuste Surveillance und die Einhaltung der Meldepflichten durch die Länder sind für eine realistische Einschätzung der Lage und die unverzügliche Einleitung der erforderlichen Maßnahmen unverzichtbar. Ein zeitnaher Informations- und Virentausch, effiziente Eindämmungsmaßnahmen weltweit und die Verständigung über Impfstrategien bestimmen über den Erfolg der Pandemiebekämpfung. Entscheidungen, die anderswo auf der Welt getroffen werden, können sich unmittelbar auch in Deutschland auswirken. Eine kontinuierliche Abstimmung über Strategien und Maßnahmen ist daher erforderlich.

In der Pandemie ist der Handlungsspielraum der Gesundheitspolitik einerseits durch die Verfassungs- und Rechtsordnung vorgegeben, andererseits aber durch die faktischen Gegebenheiten: Wie frühzeitig wird ein pandemisches Geschehen erkannt? Wie gefährlich ist der Erreger? Gelingt es und wie lange gelingt es, die Ausbreitung einzudämmen? Ist die Erkrankung therapierbar, gibt es eine Impfung? Welche Ressourcen stehen für die Bewältigung der Pandemie zur Verfügung oder können bereitgestellt werden?

\section{Die Strategie zur Pandemiebekämpfung ruht auf vier Säulen:}

1. der Überwachung der Ausbreitung, der Krankheitsverläufe und der Einleitung bevölkerungsbezogener und individueller seuchenhygienischer Maßnahmen;

2. der Sicherstellung der Versorgung, insbesondere mit antiviralen Arzneimitteln um den Zeitraum überbrücken zu können, bis Impfstoffe zur Verfügung stehen;

3. der Information der Öffentlichkeit und der Fachöffentlichkeit;

4. dem Schutz der Bevölkerung durch Impfungen.

Die Elemente der Strategie sind durch fachliche Notwendigkeiten vorgegeben, die aus dem Ziel folgen, die Gesundheit der Bevölkerung bestmöglich zu schützen. Die Aufgabenteilung und das Zusammenspiel von Bund und Ländern im Rahmen dieser Strategie und in der Kooperation mit weiteren Beteiligten wird 
durch die Rechtsordnung bestimmt. Die hierdurch vorgegebene Aufgabenteilung stellt die Akteure sowohl in der Vorbereitung auf eine Pandemie, als auch in der Pandemie, vor ganz besondere Herausforderungen und bringt nicht nur einen erheblichen Koordinierungsbedarf mit sich, sondern birgt auch das Risiko, dass zeitkritische Entscheidungen verzögert werden. Vor diesem Hintergrund wurde bereits in der Vergangenheit wiederholt eine Kompetenzverlagerung auf die Bundesebene diskutiert und wird auch Gegenstand der Diskussionen anlässlich dieser Veranstaltung sein.

Dem will ich nicht vorgreifen, sondern den Blick auf einige Beispiele lenken, die die Notwendigkeit des engen Zusammenwirkens der Akteure in Bund und Ländern in der aktuellen Pandemie verdeutlichen.

\section{Überwachung des Infektionsgeschehens und seuchenhygienische Maßnahmen:}

Bereits eine Woche nach Bekanntwerden der ersten Infektionen, am 3. Mai 2009, trat eine Rechtsverordnung der Bundesregierung in Kraft, mit der die Arztmeldepflicht nach dem Infektionsschutzgesetz auf Verdachts-, Krankheits-, und Todesfälle der neuen Influenza ausgedehnt wurde. Damit wurden die Möglichkeiten des öffentlichen Gesundheitsdienstes in den Ländern gestärkt, dem Auftreten des neuen Erregers in Deutschland so früh wie möglich mit Maßnahmen zur Krankheitsverhütung und -bekämpfung begegnen zu können.

Die Diagnostik des neuen Virus war im Nationalen Referenzzentrum für Influenza im Robert Koch-Institut (RKI) nach wenigen Tagen möglich. Dadurch und durch den Austausch in der EU und auf der Grundlage der Internationalen Gesundheitsvorschriften weltweit konnte sehr rasch ein verlässlicheres Bild über die Pandemie und ihren Verlauf gewonnen werden.

In der Frühphase der Pandemie war die Strategie der Nachverfolgung und Isolation möglichst aller Kontaktpersonen sinnvoll und erfolgreich. Sie hat die Ressourcen im öffentlichen Gesundheitsdienst der Länder stark gefordert, gemeinsam mit den günstigen Witterungsbedingungen im Sommer aber entscheidend mit dazu beigetragen, die Verbreitung der Neuen Grippe in Deutschland zu verzögern - anders als beispielsweise in England oder Spanien.

Zum Herbst hin wurde die Strategie der Nachverfolgung aufgrund steigender Fallzahlen und relativ mildem Krankheitsbild angepasst - so wurde z. B. die Arztmeldepflicht auf Todesfälle an der Neuen Influenza zurückgeführt.

Alle seuchenhygienischen Maßnahmen erfolgten auf der Grundlage des Infektionsschutzgesetzes und der durch das RKI erarbeiteten Empfehlungen u.a. zum Umgang mit Erkrankten, deren Kontaktpersonen, infizierten Reiserückkehrern 
und zur Schließung von Schulen. Die zuständigen Stellen in Bund und Ländern haben sich in dieser kritischen Phase eng - zum Teil in täglichen Telefonschaltkonferenzen - über die zu ergreifenden Maßnahmen abgestimmt.

\section{Versorgung der Bevölkerung mit antiviralen Arzneimitteln}

Die Versorgung der Bevölkerung mit antiviralen Arzneimitteln war in der aktuellen, moderaten Pandemie in Deutschland durchgehend sichergestellt. Die Länder haben hier bekanntlich Vorsorge getroffen und auch der Bund wäre in der Lage, nötigenfalls ergänzend im Wege der Katastrophenhilfe, antivirale Arzneimittel zur Verfügung zu stellen. Soweit vorübergehend das Fehlen von Säften für Kinder gemeldet wurde, standen Alternativen zur Verfügung. Problematischer ist die potentielle Engpassressource Beatmungsbetten; auch insoweit erreichten uns auch in der aktuellen moderaten Pandemie vereinzelt Meldungen über eine regional sehr hohe Auslastung.

\section{Information von Öffentlichkeit und Fachöffentlichkeit}

In ihrer Bedeutung für die Bewältigung einer Pandemie kaum zu überschätzen ist die Information von Öffentlichkeit und Fachöffentlichkeit, die dritte Säule der Strategie:

Eine zentrale Rolle sowohl bei der Information der allgemeinen Öffentlichkeit als auch insbesondere der Fachöffentlichkeit spielen die wissenschaftlichen Fachbehörden im Geschäftsbereich des BMG, das RKI und soweit Impfstoffe betroffen sind das PEI sowie die Gesundheitsbehörden der Länder. Die Fachbehörden des Bundes haben umfangreiche Informationsmaterialien und Handreichungen, insbesondere für Ärztinnen und Ärzte sowie ein umfassendes Informationsangebot im Internet bereitgestellt und eine regelmäßige Information der Presse durchgeführt.

Auch das BMG hat gleich zu Beginn der Pandemie eine Hotline eingerichtet und auf seiner Homepage ein umfassendes Informationsangebot zur Verfügung gestellt, das im Lichte des Informationsbedarfs der Bevölkerung kontinuierlich aktualisiert wurde.

Zum Schwerpunkt Hygiene haben RKI und Bundeszentrale für Gesundheitliche Aufklärung (BZgA) die Kampagne "Wir-gegen-Viren" entwickelt; unmittelbar nach Auftreten des pandemischen Virus konnte mit der Verbreitung von Informationen zum Infektionsschutz durch persönliche Hygiene begonnen werden. Die BZgA hat ergänzend eine Reihe von Informationsmaterialien zur persönli- 
chen Hygiene für Schulen entwickelt. Spots zum Händewaschen und zum richtigen Niesen komplettieren das Informationsangebot.

Auch zur Impfung wurden Informationen für die allgemeine Bevölkerung, die Zielgruppen der Empfehlung der Ständigen Impfkommission (STIKO) und das medizinische Fachpersonal erstellt und verbreitet. Informationsmaterial für die Bevölkerung und die Zielgruppen der Impfung wurde auch den Krankenkassen zur Information ihrer Versicherten zur Verfügung gestellt. Dabei wurde von Beginn an großer Wert darauf gelegt, transparent zu kommunizieren, dass die Organisation der Impfungen Aufgabe der Länder ist, dass diese länderspezifisch etwa über Beginn und Ort der Impfungen informieren und den Bürgerinnen und Bürgern mitteilen, wohin sie sich bei Nachfragen wenden können.

Ein Problem in der Bewältigung der aktuellen Pandemie bildete u. a. die divergente Diskussion in der Fachöffentlichkeit, die sich zunächst vor allem um Finanzierungsfragen rankte und im Sommer zu einem ersten „Gipfeltreffen“ im BMG u. a. mit dem Spitzenverband Bund der gesetzlichen Krankenkassen führte. Später kam die Diskussion über die Sicherheit der Impfstoffe. Letztere setzte verstärkt mit Anlaufen der Impfungen ein, die zeitlich fast mit dem Anlaufen der Herbstwelle der Influenza zusammentraf und führte zu einer deutlichen Verunsicherung in der Bevölkerung.

Man wird sich davor hüten müssen, monokausale Zusammenhänge herzustellen, die Umfragen zeigen jedoch eine Korrelation zwischen der im Vergleich zum Sommer rückläufigen Impfbereitschaft und der Diskussion über die Sicherheit der Impfstoffe. Angesichts des klaren Votums der Europäischen Zulassungsbehörden, deren Vertreterin auch im Rahmen einer im BMG veranstalteten Pressekonferenz nochmals sehr deutlich zum Ausdruck gebracht hat, dass der Nutzen der Impfung die Risiken überwiegt, angesichts der sehr transparenten Informationspolitik auch des Paul-Ehrlich-Instituts (PEI) und der STIKO, muss uns das beunruhigen. Es ist allerdings weniger ein Problem der Rechtsordnung als des Vertrauens in die Institutionen.

\section{Impfung}

Fest steht, die Impfung ist ein unverzichtbares Instrument der Pandemiebekämpfung. Bund und Länder haben daher im Rahmen der Pandemieplanung die Voraussetzungen dafür geschaffen, dass in einer - durch welches Virus auch immer ausgelösten - Pandemie der Bevölkerung ein Impfschutz angeboten werden kann.

Dabei waren für die politisch Verantwortlichen sowohl beim Abschluss der Bereitstellungsverträge als auch bei der Entscheidung über die Bestellmenge zwei Umstände ganz maßgeblich: Zum einen, dass der für die Entwicklung und 
die Produktion eines Impfstoffs zur Verfügung stehende Zeitraum deutlich kürzer sein würde, als der für die Entwicklung des üblichen saisonalen Impfstoffs; und zum anderen, dass die Produktionskapazitäten weltweit begrenzt sind.

Selbstverständlich flossen weitere Erwägungen in die Entscheidungen ein, aber diese beiden Faktoren, die angesichts des milden Verlaufs der aktuellen Pandemie aus dem Blick geraten könnten, waren maßgeblich für die schon in den Bereitstellungsverträgen getroffene Entscheidung für einen adjuvantierten Impfstoff, die Entscheidung für 10er Gebinde, ebenso wie später die Entscheidung, nicht die gesamte Bestellmenge abzunehmen, sondern zunächst für die am stärksten gefährdeten Gruppen zu bestellen.

Die Problematik wird plastisch, wenn man sich den Hinweis der WHO aus dem Frühsommer letzten Jahres in Erinnerung ruft, dass der weltweite Bedarf an Impfstoffen das Angebot übersteigen werde und den Appell der Generaldirektorin der WHO an die Staatengemeinschaft darauf eine Antwort zu geben, die auch weniger entwickelten Staaten einen, wenn auch begrenzten, Zugang zu Impfstoffen ermöglicht. Deutschland hat eine Antwort auch auf diese Herausforderung gegeben und international und auf europäischer Ebene nachdrücklich für diesen Weg geworben. 
